\title{
Probing lepton flavour violation in slepton NLSP scenarios
}

\section{Koichi Hamaguchi}

Deutsches Elektronen-Synchrotron, DESY

22603 Hamburg, Germany

E-mail: koichi.hamaguchi@desy.de

\section{Alejandro Ibarra}

Department of Physics, Theory Division, CERN

CH-1211 Geneva 23, Switzerland, and

Instituto de Física Teórica, CSIC/UAM, C-XVI, Universidad Autónoma de Madrid

Cantoblanco, 28049 Madrid, Spain

E-mail: alejandro.ibarra@cern.ch

ABSTRACT: In supersymmetric models where the gravitino is the lightest superparticle, the next-to-lightest superparticle (NLSP) is long-lived, and hence could be collected and studied in detail. We study the prospects of direct detection of lepton flavour violation in charged slepton NLSP decays. Mixing angles in the slepton sector as small as $\sim 3 \times 10^{-2}$ $\left(9 \times 10^{-3}\right)$ could be probed at the $90 \%$ confidence level if $3 \times 10^{3}\left(3 \times 10^{4}\right)$ sleptons could be collected.

KeYwords: Supersymmetry Phenomenology, Supergravity Models, Supersymmetric Standard Model, Rare Decays. 


\section{Contents}

1. Introduction 1

2. Charged slepton mass spectrum and decays 2

3. Possible scenarios 7

4. Conclusions 13

A. Electron spectrum from tau decay 13

B. Probing $R$-parity violation in slepton NLSP scenarios 15

\section{Introduction}

In a supersymmetric (SUSY) theory with R-parity conserved the lightest superparticle (LSP) is stable. This property puts forward the LSP as a natural candidate for the dark matter of the Universe, and several candidates have been proposed in the literature: neutralinos, axinos, gravitinos... In this paper we concentrate on the darkest among all the dark matter candidates, the gravitino, which in the early universe can be produced by thermal scatterings [1] and in the decays of superparticles [2].

If the gravitino is the LSP, the next-to-lightest superparticles (NLSPs) can only decay to gravitinos and other Standard Model particles via gravitational interactions. As a consequence, NLSPs are very long lived and could be collected and studied with detail in future experiments. Recently, prospects of collecting charged NLSPs and detecting their decay products in future colliders have been discussed [3, 1 . At the LHC, cascade decays of squarks and gluinos can produce of the order of $10^{6}$ NLSPs per year if the sparticle masses are close to the current experimental limits [5. Among them $\mathcal{O}\left(10^{3}-10^{4}\right)$ NLSPs could be collected by placing 1-10 kton massive material around the LHC detectors. On the other hand, at the Linear Collider [6] up to $\mathcal{O}\left(10^{3}-10^{5}\right)$ NLSPs may be collected and studied. If the stopper material simultaneously serves as an active real-time detector [3, the decay products and their distributions could be studied in detail there.

If a sample of NLSPs could be accumulated, their decays could be observed and studied without important backgrounds, allowing a precise determination of the supersymmetric parameters. In ref. [7], NLSPs decays were used as a method to prove the existence of supergravity in nature. Measurement of the NLSP lifetime, together with kinematically reconstructed gravitino mass, could lead to a microscopic determination of the Planck scale. Moreover, study of a rare three-body decay could reveal the peculiar couplings of gravitino, and may even determine the gravitino spin. 
Future colliders will open new opportunities to detect lepton flavour violation (LFV), complementary to the ongoing and projected experiments on rare decays. There is mounting literature on the prospects to detect LFV in the LHC or in the future Linear Collider [8, under the implicit assumption that the neutralino is the LSP. In this paper we assume that the gravitino is the LSP and the NLSP is a charged slepton, and study the prospects of direct detection of lepton flavour violation in their decays. The basic idea is very simple. Suppose that many NLSP sleptons could be collected, say staus. If there is no LFV, all of them would decay into taus and gravitinos, $\widetilde{\tau} \rightarrow \tau \psi_{3 / 2}$. If on the contrary LFV exists in nature, some of the collected staus would decay as $\widetilde{\tau} \rightarrow e \psi_{3 / 2}$ or $\widetilde{\tau} \rightarrow \mu \psi_{3 / 2}$, which would constitute a direct signal of LFV.

Different scenarios will arise depending on the spectrum of charged sleptons and on their different decay modes. This is discussed in detail in section 2. The signals for lepton flavour violation in NLSP decay are systematically studied for the different scenarios in section 3. Our conclusions are presented in section 4 Finally, we include two appendices with a review of the electron spectrum from the decay of taus in flight, and some comments about the implementation of this idea for the case with R-parity violation.

\section{Charged slepton mass spectrum and decays}

The charged slepton masses receive contributions from the supersymmetric lagrangian and from the soft breaking terms. The supersymmetric contributions include those from the superpotential, that are related to the charged lepton masses, and the D-terms. In the minimal scenario, the D-term contribution is proportional to the $Z$ mass and $\tan \beta$, although in more general scenarios there could be additional contributions coming from the breaking of extra gauge groups at intermediate energies, that could be flavour conserving or flavour violating [9]. In addition to these, there is a contribution to the charged slepton masses from the SUSY soft breaking terms:

$$
-\mathcal{L}_{\text {soft }}=\left(m_{\tilde{l}_{L}}^{2}\right)_{i j} \widetilde{l}_{L_{i}}^{\dagger} \widetilde{l}_{L_{j}}+\left(m_{\tilde{l}_{R}}^{2}\right)_{i j} \widetilde{l}_{R_{i}}^{\dagger} \widetilde{l}_{R_{j}}+\left(Y_{i j}^{l} A_{l i j} \widetilde{l}_{R_{i}} \widetilde{l}_{L_{j}} H_{d}+\text { h.c. }\right),
$$

where $i, j=1,2,3$ are generational indices. $\widetilde{l}_{L, R}$ denote the left and right-handed charged sleptons, $H_{d}$ is the down-type Higgs doublet, $Y_{i j}^{l}$ is the charged-lepton Yukawa coupling, and $\left(m_{\tilde{l}_{L, R}}^{2}\right)_{i j}$ and $A_{l i j}$ are the soft scalar mass matrix squared and the soft trilinear matrix, respectively. This contribution is in general flavour violating and mixes left-handed and right-handed sleptons through the trilinear terms.

The resulting $6 \times 6$ mass matrix reads:

$$
\mathcal{M}^{2}=\left(\begin{array}{cc}
m_{\tilde{l}_{L}}^{2}+m_{l}^{2}-\left(\frac{1}{2}-\sin ^{2} \theta_{W}\right) m_{Z}^{2} \cos 2 \beta & m_{l}\left(A_{l}-\mu \tan \beta\right) \\
m_{l}\left(A_{l}-\mu \tan \beta\right) & m_{\tilde{l}_{R}}^{2}+m_{l}^{2}-\sin ^{2} \theta_{W} m_{Z}^{2} \cos 2 \beta
\end{array}\right),
$$

which is diagonalized by certain mass eigenstates, $\widetilde{l}_{\alpha}$. We will label the mass eigenstates so that $\widetilde{l}_{1}$ is the lightest and $\widetilde{l}_{6}$ the heaviest. The mass eigenstates are related to the flavour eigenstates by the unitary transformation

$$
\widetilde{l}_{\alpha}=U_{\alpha, i} \widetilde{l}_{L_{i}}+U_{\alpha, i+3} \widetilde{l}_{R_{i}}
$$


where $\alpha=1, \ldots, 6$ runs over the mass eigenstates, and $i=1,2,3$ runs over the flavour eigenstates $(\mathrm{e}, \mu, \tau)$. For instance, the matrix element $U_{\alpha, 3}$ measures the $\widetilde{\tau}_{L}$ content of the mass eigenstate $\widetilde{l}_{\alpha}$, whereas $U_{\alpha, 6}$ indicates the $\widetilde{\tau}_{R}$ content.

If flavour violation is not very large in nature, as present experiments suggest, then the mass eigenstates are mainly a combination of left and right-handed sfermions of the same generation. We expect that the lightest charged slepton is mainly a combination of staus, as a result of the effect of the trilinear terms and of the negative contribution to the slepton masses from the tau radiative corrections. We will assume this in what follows, although our results can be generalized to the other possibilities.

From the mass matrix, it is apparent that when the left and right-handed soft scalar masses are approximately equal at high energies, $m_{\tilde{l}_{L}}^{2} \simeq m_{\tilde{l}_{R}}^{2}$, the right-handed sfermions are going to be lighter than their left-handed counterparts, due to the D-term contribution to the scalar masses. There is however a more important effect that splits left and right handed sleptons, namely radiative corrections. Left-handed sfermion masses receive a positive contribution at low energies from the wino radiative corrections, whereas the righthanded ones do not. Therefore, in a scenario where at high energies the left-handed and right-handed soft masses are very similar, the lightest charged slepton will approximately correspond to the right-handed stau, i.e., $\widetilde{l}_{1} \approx \widetilde{\tau}_{R}$. Nevertheless, when $\tan \beta$ is large, $\widetilde{l}_{1}$ can have a non-negligible component of left-handed stau, because of the enhancement of left-right mixing at large $\tan \beta$, that otherwise is suppressed by the small tau mass. In smuon and selectron sectors left-right mixings are very small, and the (next-to-) next-tolightest charged slepton will almost correspond to the right-handed smuon (selectron), i.e., $\widetilde{l}_{2}, \widetilde{l}_{3} \approx \widetilde{\mu}_{R}, \widetilde{e}_{R}$.

In the presence of lepton flavour violations the mass eigenstates are in general linear combinations of all the flavour eigenstates. For simplicity, in the following the mass eigenstates will be sometimes referred by the closest flavour eigenstates. For instance, $m_{\widetilde{\tau}}$ will represent the mass of $\widetilde{l}_{1}$, which is mainly the stau $\widetilde{\tau}$.

After discussing qualitatively the features of the charged slepton spectrum, let us study their decay modes in the scenario where the gravitino is the LSP. Charged sleptons can decay via gravitational interactions to a gravitino and a charged lepton. If the lightest charged slepton is the NLSP, this is the only possible decay channel. On the other hand, heavier charged sleptons can also decay to NLSPs and neutrinos via chargino exchange $\widetilde{l}_{2}^{-} \rightarrow \widetilde{l}_{1}^{-} \bar{\nu}_{i} \nu_{j}$, or to NLSPs and charged leptons via neutralino exchange $\widetilde{l}_{2}^{-} \rightarrow \widetilde{l}_{1}^{-} \bar{l}_{i}^{ \pm} l_{j}^{\mp}$ (and analogously for the positively charged sleptons). These processes and their decay rates were studied in detail in [10].

The decay of a charged slepton mass eigenstate into a gravitino and a charged lepton is described by the following lagrangian [11]:

$$
\mathcal{L}_{3 / 2}=-\frac{1}{\sqrt{2} M_{P}}\left[U_{\alpha, i}^{*}\left(D_{\nu} \widetilde{l}_{\alpha}\right) \bar{l}_{i} P_{R} \gamma_{\mu} \gamma^{\nu} \psi^{\mu}+U_{\alpha, i+3}^{*}\left(D_{\nu} \widetilde{l}_{\alpha}\right) \bar{l}_{i} P_{L} \gamma_{\mu} \gamma^{\nu} \psi^{\mu}+\text { h.c. }\right]
$$

where $D_{\nu} \widetilde{l}_{\alpha}=\left(\partial_{\nu}+i e A_{\nu}\right) \widetilde{l}_{\alpha}$ is the covariant derivative and $M_{P}=\left(8 \pi G_{N}\right)^{-1 / 2}$ is the reduced Planck mass. From this lagrangian, it is straightforward to compute the total 
decay rate of the slepton $\widetilde{l}_{\alpha}$ into a lepton with flavour $i$ and a gravitino. The result is

$$
\begin{aligned}
\Gamma\left(\widetilde{l}_{\alpha} \rightarrow l_{i} \psi_{3 / 2}\right)= & \frac{m_{\tilde{l}_{\alpha}}^{5}}{48 \pi m_{3 / 2}^{2} M_{P}^{2}}\left(\left|U_{\alpha, i}\right|^{2}+\left|U_{\alpha, i+3}\right|^{2}\right)\left(1-\frac{m_{3 / 2}^{2}+m_{l_{i}}^{2}}{m_{\tilde{l}_{\alpha}}^{2}}\right)^{4} \times \\
& \times\left(1-\frac{4 m_{3 / 2}^{2} m_{l_{i}}^{2}}{\left(m_{\tilde{l}_{\alpha}}^{2}-m_{3 / 2}^{2}-m_{l_{i}}^{2}\right)^{2}}\right)^{3 / 2} \times \\
& \times\left(1-\frac{4 m_{3 / 2} m_{l_{i}}}{m_{\tilde{l}_{\alpha}}^{2}-m_{3 / 2}^{2}-m_{l_{i}}^{2}} \cdot \frac{\operatorname{Re} U_{\alpha, i} U_{\alpha, i+3}^{*}}{\left|U_{\alpha, i}\right|^{2}+\left|U_{\alpha, i+3}\right|^{2}}\right) \\
\simeq & \frac{m_{\tilde{l}_{\alpha}}^{5}}{48 \pi m_{3 / 2}^{2} M_{P}^{2}}\left(\left|U_{\alpha, i}\right|^{2}+\left|U_{\alpha, i+3}\right|^{2}\right)\left(1-\frac{m_{3 / 2}^{2}}{m_{\tilde{l}_{\alpha}}^{2}}\right)^{4},
\end{aligned}
$$

where in the last step we have neglected the mass of the lepton. The decay rates are typically of the order of $10^{-22} \mathrm{GeV}$ to $10^{-33} \mathrm{GeV}$ for gravitino masses of $\sim 1 \mathrm{MeV}$ to $\sim 100 \mathrm{GeV}$, and NLSP masses of $\sim 150 \mathrm{GeV}$. In the case of the lightest charged slepton, that can only decay to a gravitino, these decay rates translate into lifetimes of the order of mili-seconds to years, respectively.

The key idea of the present analysis comes from the fact that these decays could have a non-trivial flavour structure, that is reflected in the $6 \times 6$ unitary matrix $U$. Therefore, one could directly measure the slepton mixings by studying the branching ratios:

$$
\left|U_{\alpha, i}\right|^{2}+\left|U_{\alpha, i+3}\right|^{2} \propto B R\left(\widetilde{l}_{\alpha} \rightarrow l_{i} \psi_{3 / 2}\right)
$$

Especially,

$$
\left|U_{1, i}\right|^{2}+\left|U_{1, i+3}\right|^{2} \simeq B R\left(\widetilde{l}_{1} \rightarrow l_{i} \psi_{3 / 2}\right) .
$$

For this to be feasible, it would be desirable that all the heavier sleptons decay fast enough into the NLSP. ${ }^{1}$ Otherwise, the stopping material would be polluted with nextto-NLSPs (NNLSPs), whose decays could represent sources of background for the lepton flavour violating decays that we want to study. We will show that if lepton flavour violation is large enough to be observed experimentally, it is guaranteed that this situation will not happen, allowing a clean observation of the LFV effects.

For the sake of the presentation, let us first discuss the decay modes in the case without flavour violation. The dominant decay channel is normally the one mediated by neutralinos, provided that the decay is kinematically accessible. This requires a mass difference between the NLSP and the NNLSP larger than the tau mass, that despite being very small is not always guaranteed. For instance, radiative corrections induced by the tau generate a mass

\footnotetext{
${ }^{1}$ In the following we will assume that neutralinos are heavier than the next-to-lightest charged sleptons $\widetilde{l}_{2}, \widetilde{l}_{3} \approx \widetilde{\mu}_{R}, \widetilde{e}_{R}$. If on the contrary the lightest neutralino $\widetilde{N}_{1}$ is lighter than the selectron (smuon), i.e., $m_{\tilde{\tau}}<m_{\widetilde{N}_{1}}<m_{\tilde{e}_{R}}\left(m_{\tilde{\mu}_{R}}\right)$, the $\widetilde{e}_{R}\left(\widetilde{\mu}_{R}\right)$ mainly decays into $\widetilde{N}_{1}$, not into $\widetilde{\tau}$. However, as far as $m_{\tilde{\tau}}+m_{\tau}<$ $m_{\widetilde{N}_{1}}<m_{\tilde{e}_{R}}\left(m_{\widetilde{\mu}_{R}}\right)$, the neutralino $\widetilde{N}_{1}$ quickly decays into $\widetilde{\tau}$ and hence reduces to the cases discussed in the text. If the neutralino mass happens to lie in the narrow range $m_{\widetilde{\tau}}<m_{\tilde{N}_{1}}<m_{\tilde{\tau}}+m_{\tau}, \tilde{N}_{1}$ can have a long lifetime and may escape from the stopper material.
} 
difference which is $m_{\widetilde{l}_{2}}-m_{\widetilde{l}_{1}} \sim 10^{-2} m_{\tau} \tan ^{2} \beta$, that is large enough when $\tan \beta$ is large. Moreover, left-right mixing also produces a mass splitting between the NLSP and the NNLSP, that reads:

$$
m_{\widetilde{l}_{2}}-m_{\widetilde{l}_{1}} \simeq \frac{m_{\tau}^{2}\left(A_{\tau}-\mu \tan \beta\right)^{2}}{2 m_{\widetilde{l}_{1}}\left(m_{\widetilde{l}_{L}}^{2}-m_{\widetilde{l}_{R}}^{2}+\left(2 \sin ^{2} \theta_{W}-\frac{1}{2}\right) m_{Z}^{2} \cos 2 \beta\right)}
$$

Hence, $m_{\widetilde{l}_{2}}-m_{\widetilde{l}_{1}}>m_{\tau}$ is obtained when $\left|A_{\tau}-\mu \tan \beta\right| \gtrsim \sqrt{2 m_{\widetilde{l}_{1}}\left(m_{\widetilde{l}_{L}}^{2}-m_{\widetilde{l}_{R}}^{2}\right) / m_{\tau}}$, that is fulfilled for some values of $A_{\tau}$, or for large values of $\tan \beta$.

Therefore, when $\tan \beta$ is large, generically larger than $\sim 10$, the mass difference between the NLSP and the NNLSP is large enough to allow the decay via neutralino exchange. On the other hand, when $\tan \beta$ is small, there are regions of the parameter space where this mass difference is not large enough and the NNLSP can only decay into the NLSP via chargino exchange. ${ }^{2}$ The latter decay channel is suppressed by small Yukawa couplings, resulting in very small decay rates. In particular, the decay rate of the right-handed selectron via chargino interaction can be so small, that the decay into gravitinos could become the dominant channel. If this happens, the sample would be polluted with right-handed selectrons, and the electrons from their flavour conserving decay $\widetilde{e} \rightarrow e \psi_{3 / 2}$ could mask the electrons from the flavour violating decay of the staus $\widetilde{\tau} \rightarrow e \psi_{3 / 2}$. The decay rates for the NNLSP and the NNNLSP are illustrated in figure 1 for a specific choice of SUSY parameters compatible with cosmology [12]. In the plot, we have omitted the contribution from the decays into gravitino. We assume the boundary conditions for the constrained MSSM, and we run the renormalization group equations to low energies to obtain the mass spectrum. As usual, the $\mu$ parameter is determined by imposing a correct electroweak symmetry breaking. For $\tan \beta=3$, the decay of smuon (selectron) into stau, tau and a charged lepton via neutralino exchange is kinematically forbidden for $A_{0} \gtrsim-650 \mathrm{GeV}$, and the decay rates become suppressed. For larger $\tan \beta$, the decay into tau is open and the rates become large.

It is interesting to note that if flavour violation exists in nature this picture changes radically. The reason for this is double. First, flavour violation in the right-handed sector can induce a non-degeneracy among the mass eigenstates, that can be large enough to open kinematically the (fast) decay channel mediated by neutralinos. To be precise, this occurs when $\left(m_{\widetilde{l}_{R}}^{2}\right)_{13} / m_{\widetilde{l}_{1}} \gtrsim m_{\tau}$. Analogously, some amount of flavour violation in the righthanded smuon-stau sector is enough to open kinematically the decay $\widetilde{\mu} \rightarrow \widetilde{\tau} \mu \tau$. Notice that $\left(m_{\widetilde{l}_{R}}^{2}\right)_{23} / m_{\widetilde{l}_{1}} \gtrsim m_{\tau}$ is not in conflict with the present bound $B R(\tau \rightarrow \mu \gamma) \lesssim 6 \times 10^{-7}$, that requires $\left(m_{\widetilde{l}_{R}}^{2}\right)_{23} / m_{\widetilde{l}_{1}} \lesssim 100 \mathrm{GeV}$ for $\tan \beta \simeq 3$ and $m_{\widetilde{l}_{1}} \simeq 150 \mathrm{GeV} 13$.

There is nevertheless a more important effect of flavour violation. If flavour violation exists in nature, even in very small amounts, the flavour violating decay channel mediated by neutralinos $\widetilde{e} \rightarrow \widetilde{\tau} e$ e can be very efficient. Despite the decay rate is suppressed by

\footnotetext{
${ }^{2}$ We will neglect the 4- and 5-body decay modes via virtual tau exchange, $\widetilde{e} \rightarrow \widetilde{\tau} e \bar{\nu}_{\tau} X$ where $X$ is $e \bar{\nu}_{e}$, $\mu \bar{\nu}_{\mu}, \pi^{-}$and so on. The rates of those modes may become comparable to $\Gamma\left(\widetilde{e} \rightarrow \widetilde{\tau} \bar{\nu}_{\tau} \nu_{e}\right)$. In such cases, $\Gamma\left(\widetilde{e} \rightarrow \widetilde{\tau} \bar{\nu}_{\tau} \nu_{e}\right)$ should be replaced with $\Gamma\left(\widetilde{e} \rightarrow \widetilde{\tau} \bar{\nu}_{\tau} \nu_{e}\right)+\Gamma\left(\widetilde{e} \rightarrow \widetilde{\tau} e \bar{\nu}_{\tau} X\right)$ in the following discussion, but our conclusions are not affected.
} 


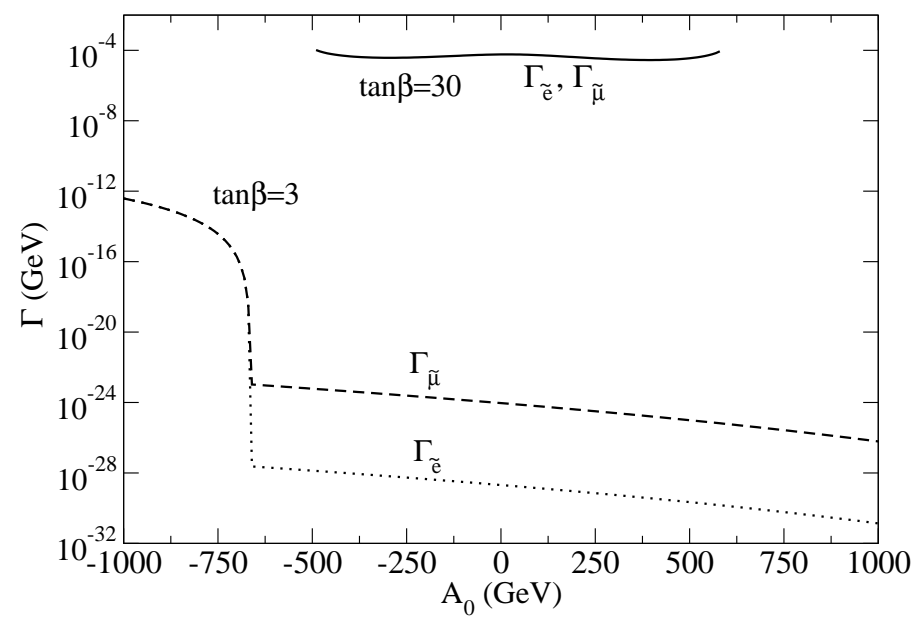

Figure 1: Decay rates for the selectron and the smuon for specific choices of SUSY parameters in the constrained MSSM scenario, namely, $m_{0}=0, M_{1 / 2}=400 \mathrm{GeV}, \mu>0$, for the case without lepton flavour violation. These parameters lead to charged slepton masses of $m_{\widetilde{l}_{1}} \approx 150 \mathrm{GeV}$ for $\tan \beta=3$ and $m_{\tilde{l}_{1}} \approx 50-100 \mathrm{GeV}$ for $\tan \beta=30$. For large $\tan \beta$ and large $\left|A_{0}\right|$ the spectrum presents tachyonic sleptons.

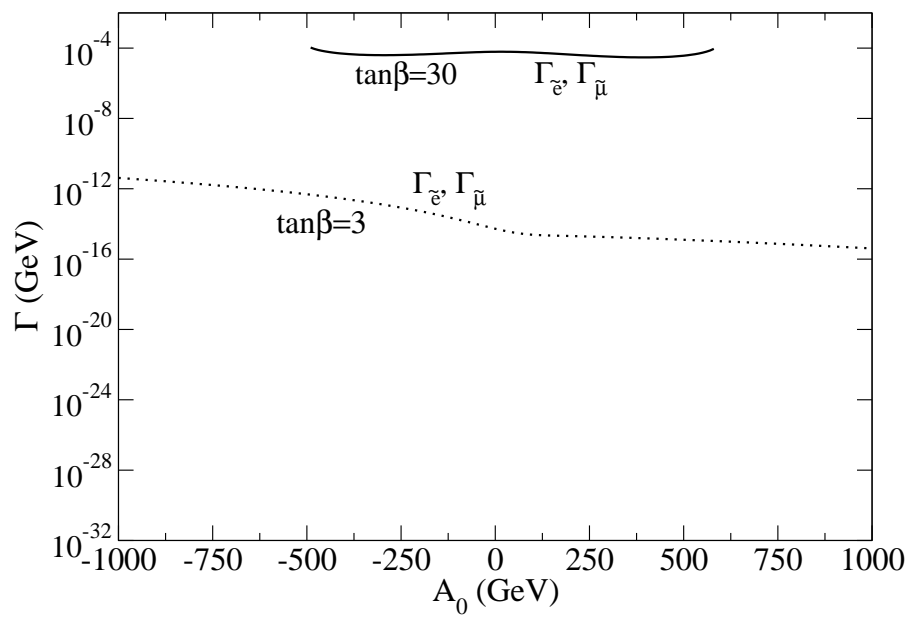

Figure 2: The same as figure 1 with a small flavour violation in the right-handed slepton mass matrix: $\left(m_{\tilde{l}_{R}}^{2}\right)_{23} / m_{\tilde{l}_{1}}^{2}=\left(m_{l_{R}}^{2}\right)_{13} / m_{\tilde{l}_{1}}^{2}=0.01$.

the small mixing angle, it could be orders of magnitude larger than the decay rates of the processes mediated by charginos or into gravitinos. Notice also that this decay channel is usually kinematically open, since radiative corrections already induce a mass splitting between the selectron and the stau larger than twice the electron mass. In figure 2, we show the effect of flavour violation on the decay rates of the smuon and the selectron. It is remarkable the double role that lepton flavour violation plays in our analysis: it is not only the object of our investigation, but also a crucial ingredient for the preparation of a sample with just NLSPs, that would allow a clean detection of the flavour violation itself. 


\section{Possible scenarios}

Whether the sample contains just NLSPs or on the contrary also contains (N)NNLSPs depends on the relative size of the different decay rates (gravitational and mediated by charginos and neutralinos). Let us consider the case in which only two species are relevant, namely staus and selectrons. We assume that smuons are not present in the sample, since in general these will decay fast enough into staus. Notice from figure 1 that even the decay by chargino exchange, the most inefficient one apart from the gravitational channel, is generically orders of magnitude larger than the decay into gravitinos. ${ }^{3}$ However, as we mentioned in the previous section, this is not always the case for the selectrons, whose decay rate via chargino exchange is further suppressed by the small electron Yukawa coupling.

In a sample that contains just stopped selectrons and staus, the following decay processes can occur. There are the lepton flavour conserving decays into gravitinos,

$$
\begin{aligned}
\Gamma\left(\widetilde{\tau} \rightarrow \tau \psi_{3 / 2}\right) & \equiv \Gamma_{F C}^{\widetilde{\tau}}, \\
\Gamma\left(\widetilde{e} \rightarrow e \psi_{3 / 2}\right) & \equiv \Gamma_{F C}^{\widetilde{e}} ;
\end{aligned}
$$

the decays of the selectron into staus via neutralino or chargino exchange, both the flavour conserving channel and possibly also a flavour violating channel,

$$
\begin{aligned}
\Gamma\left(\widetilde{e} \rightarrow \widetilde{\tau} \bar{\nu}_{\tau} \nu_{e}\right)+\Gamma(\widetilde{e} \rightarrow \widetilde{\tau} \tau e) & \equiv \Gamma_{F C, \widetilde{e} \tau} \\
\Gamma(\widetilde{e} \rightarrow \widetilde{\tau} e e) & \equiv \Gamma_{F V, \widetilde{e} \tau}
\end{aligned}
$$

and possibly lepton flavour violating decays into gravitinos

$$
\begin{aligned}
\Gamma\left(\widetilde{\tau} \rightarrow e \psi_{3 / 2}\right) & \equiv \Gamma_{F V, e}^{\widetilde{\tau}}, \\
\Gamma\left(\widetilde{\tau} \rightarrow \mu \psi_{3 / 2}\right) & \equiv \Gamma_{F V, \mu}^{\widetilde{\tau}}, \\
\Gamma\left(\widetilde{e} \rightarrow \tau \psi_{3 / 2}\right) & \equiv \Gamma_{F V, \tau}^{\widetilde{e}}, \\
\Gamma\left(\widetilde{e} \rightarrow \mu \psi_{3 / 2}\right) & \equiv \Gamma_{F V, \mu}^{\widetilde{e}} .
\end{aligned}
$$

Here, we have omitted the selectron-smuon conversion $\widetilde{e} \rightarrow \widetilde{\mu} \bar{\nu}_{\mu} \nu_{e}$ (or $\rightarrow \widetilde{\mu} \mu e$ ) assuming that it is much suppressed compared to the selectron-stau conversion. ${ }^{4}$

The total decay rates for the stau and the selectron read:

$$
\begin{aligned}
& \Gamma_{\text {total }}^{\widetilde{\tau}}=\Gamma_{F C}^{\widetilde{\tau}}+\Gamma_{F V}^{\widetilde{\tau}}, \\
& \Gamma_{\text {total }}^{\widetilde{e}}=\Gamma_{F C}^{\widetilde{e}}+\Gamma_{F V}^{\widetilde{e}}+\Gamma_{\widetilde{e} \tau},
\end{aligned}
$$

where we have defined $\Gamma_{\widetilde{e} \widetilde{\tau}} \equiv \Gamma_{F C, \widetilde{e} \tilde{\tau}}+\Gamma_{F V, \widetilde{e} \tau}$ as the total decay rate of conversion of selectron into stau.

\footnotetext{
${ }^{3}$ We discard the extreme case where $\Gamma\left(\widetilde{\mu} \rightarrow \widetilde{\tau} \nu_{\mu} \bar{\nu}_{\tau}\right) \sim 10^{-24} \mathrm{GeV} \lesssim \Gamma\left(\widetilde{\mu} \rightarrow \mu \psi_{3 / 2}\right)$, which can happen if $\tan \beta$ is small and $m_{3 / 2} \lesssim 3 \mathrm{MeV}\left(m_{\tilde{\mu}} / 100 \mathrm{GeV}\right)^{5 / 2}$.

${ }^{4}$ Even if $\widetilde{e} \rightarrow \widetilde{\mu} \bar{\nu}_{\mu} \nu_{e}$ (or $\rightarrow \widetilde{\mu} \mu e$ ) occurs, it is followed by a prompt decay of smuon, $\widetilde{\mu} \rightarrow \widetilde{\tau} \bar{\nu}_{\tau} \nu_{\mu}$ (or $\rightarrow \widetilde{\tau} \tau \mu$ ), and effectively the whole process plays the role of $\widetilde{e} \rightarrow \widetilde{\tau}$ conversion.
} 
The abundances of the different species are determined by the following set of differential equations:

$$
\begin{aligned}
\frac{d N_{\widetilde{\tau}}}{d t} & =-\Gamma_{\text {total }}^{\widetilde{\tau}} N_{\widetilde{\tau}}+\Gamma_{\widetilde{e} \widetilde{\tau}} N_{\widetilde{e}}, \\
\frac{d N_{\widetilde{e}}}{d t} & =-\Gamma_{\text {total }}^{\widetilde{e}} N_{\widetilde{e}}, \\
\frac{d N_{e}}{d t} & =\Gamma_{F C}^{\widetilde{e}} N_{\widetilde{e}}+\Gamma_{F V, e}^{\widetilde{\tau}} N_{\widetilde{\tau}}, \\
\frac{d N_{\tau}}{d t} & =\Gamma_{F C}^{\widetilde{\tau}} N_{\widetilde{\tau}}+\Gamma_{F V, \tau}^{\widetilde{e}} N_{\widetilde{e}}, \\
\frac{d N_{\mu}}{d t} & =\Gamma_{F V, \mu}^{\widetilde{\tau}} N_{\widetilde{\tau}}+\Gamma_{F V, \mu}^{\widetilde{e}} N_{\widetilde{e}},
\end{aligned}
$$

where $N_{e, \mu, \tau}$ are the numbers of leptons coming directly from gravitational two-body decays.

A fraction of taus will decay into muons and electrons with branching ratios $B R\left(\tau^{-} \rightarrow\right.$ $\left.\mu^{-} \bar{\nu}_{\mu} \nu_{\tau}\right)=17.36 \pm 0.06 \%$ and $B R\left(\tau^{-} \rightarrow e^{-} \bar{\nu}_{e} \nu_{\tau}\right)=17.84 \pm 0.06 \%$ [14. These decay products could mask the muons and electrons coming from the lepton flavour violating decays $\widetilde{\tau} \rightarrow \mu \psi_{3 / 2}, \widetilde{\tau} \rightarrow e \psi_{3 / 2}$, and represent a source of background. This background can be distinguished from the signal by looking at the energy spectrum: the leptons from the flavour conserving tau decay present a continuous energy spectrum, in stark contrast with the leptons coming from the two body gravitational decay, whose energies are sharply peaked at $E_{0}=\left(m_{\widetilde{\tau}, \tilde{e}}^{2}+m_{\mu, e}^{2}-m_{3 / 2}^{2}\right) /\left(2 m_{\tilde{\tau}, \tilde{e}}\right)$. It is easy to check that only a very small fraction of the electrons and muons from the tau decay have energies close to this cut-off energy. For instance, for the typical energy resolution of an electromagnetic calorimeter, $\sigma \simeq 10 \% / \sqrt{E(\mathrm{GeV})}$, only $2 \times 10^{-5}$ of the taus with energy $E_{0} \sim 100 \mathrm{GeV}$ will produce electrons with energy $\simeq E_{0}$, within the energy resolution of the detector, which could be mistaken for electrons coming from the LFV stau decay (see appendix A for details). In the following, we will include this source of background for completeness using the number $2 \times 10^{-5}$, but in many instances it is negligible. For the muon signal, however, this may be problematic since the measurement of the muon energy is experimentally difficult. For simplicity, in the following, we will concentrate on the search for $\widetilde{\tau} \rightarrow e \psi_{3 / 2}$.

Some additional comments on possible experimental setups are in order. To measure the temporal distributions of the events precisely, the stopper itself had better be at the same time an active detector [3]. It measures the time when the NLSP is stopped $\left(t_{\text {stop }}\right)$ and the time when it decays $\left(t_{\text {decay }}\right)$ for each individual NLSP. By taking a distribution of the $\left(t_{\text {decay }}-t_{\text {stop }}\right)$ for the samples, one can measure the lifetime. In the following, we will assume that $\Gamma_{\text {total }}^{\widetilde{\tau}} \simeq \Gamma\left(\widetilde{\tau} \rightarrow \tau \psi_{3 / 2}\right) \ll 10^{-17} \mathrm{GeV}$, or equivalently $\tau_{\widetilde{\tau}} \gg 100 \mathrm{nsec}$, or $c \tau_{\widetilde{\tau}} \gg 10 \mathrm{~m}$. Otherwise staus would decay inside the main detector and it would not be possible to collect them in sufficiently large amounts. This is the case if $m_{3 / 2} \gg 1 \mathrm{keV} \times\left(m_{\widetilde{\tau}} / 100 \mathrm{GeV}\right)^{5 / 2}$.

Concerning the production, there are roughly speaking two possibilities. At the LHC, the numbers of produced $\widetilde{\tau} \mathrm{s}, \widetilde{e s}$, (and $\widetilde{\mu} \mathrm{s}$ ) will be almost the same, since they are mostly generated by cascade decays of squarks and gluinos. On the other hand, at the Linear 
Collider, the numbers can be different. Especially, at the $e^{-} e^{-}$mode, suggested in [3] to produce many slow sleptons, initially only $\widetilde{e}$ are produced.

Now let us discuss possible scenarios. The total number of electrons detected can be obtained solving eqs. (3.5). After a sufficiently long observation time, and ignoring negligible backgrounds, the solution can be simplified to

$$
N_{e} \simeq N_{\widetilde{e}} B R\left(\widetilde{e} \rightarrow e \psi_{3 / 2}\right)+\left(N_{\widetilde{\tau}}+N_{\widetilde{e}} \frac{\Gamma_{\widetilde{e} \widetilde{\tau}}}{\Gamma_{\text {total }}^{\widetilde{e}}}\right) B R\left(\widetilde{\tau} \rightarrow e \psi_{3 / 2}\right) .
$$

The first term is the most important source of background, which becomes relevant when $N_{\widetilde{e}} B R\left(\widetilde{e} \rightarrow e \psi_{3 / 2}\right) \gtrsim 1$. This is going to arise only when the following three conditions occur simultaneously:

- $\widetilde{e} \rightarrow \widetilde{\tau} e \tau$ is kinematically closed, which requires $m_{\tilde{e}}-m_{\tilde{\tau}}<m_{\tau}$.

- $\Gamma(\widetilde{e} \rightarrow \widetilde{\tau} e e) \lesssim N_{\widetilde{e}} \Gamma\left(\widetilde{e} \rightarrow e \psi_{3 / 2}\right)$, which requires tiny lepton flavour violation. For instance, for the parameters of figure 1 and $\tan \beta=3, A_{0}=0$, this condition requires $\left|U_{11}\right|^{2}+\left|U_{14}\right|^{2} \lesssim 10^{-14}$ for $N_{\widetilde{e}}=10^{4}$.

- $\Gamma\left(\widetilde{e} \rightarrow \widetilde{\tau} \bar{\nu}_{\tau} \nu_{e}\right) \lesssim N_{\widetilde{e}} \Gamma\left(\widetilde{e} \rightarrow e \psi_{3 / 2}\right)$. This condition is the weakest among all. For the same parameters as above, this condition requires $m_{3 / 2} \lesssim 60 \mathrm{GeV}$ for $N_{\widetilde{e}}=10^{4}$.

When any of these three conditions fails, the backgrounds are essentially negligible, therefore any electron observed in the detector would be a clear indication for LFV. To estimate the prospects for detection of flavour violation, we will consider the cases of the LHC and the $e^{-} e^{-}$Linear Collider (LC), and we will assume that particle identification is very good in the stopper-detector. The temporal distribution of electrons is shown in figure 3 for different amounts of lepton flavour violation. As typical numbers for the LHC, we take $N_{\widetilde{\tau}}$ (init.) $=N_{\widetilde{\mu}}$ (init.) $=N_{\widetilde{e}}$ (init.) $=1000$, so that after the selectrons and smuons have decayed, the sample consists of 3000 staus. The total number of electrons observed over the whole observation time is $273,29.7,3.0$ and 0.06 for $\Gamma\left(\widetilde{\tau} \rightarrow e \psi_{3 / 2}\right) / \Gamma(\widetilde{\tau} \rightarrow$ $\left.\tau \psi_{3 / 2}\right)=0.1,0.01,0.001$ and 0 , respectively. On the other hand, for the $\mathrm{LC}$ we take as a representative possibility $N_{\widetilde{\tau}}$ (init.) $=0, N_{\widetilde{\mu}}$ (init.) $=0, N_{\widetilde{e}}$ (init.) $=30000$, which lead to $N_{\tilde{\tau}}=30000$ after selectrons decay. In this case, the number of events are 2727, 297, 30.0 and 0.6 (notice that the number of events from the background is always smaller than one).

Inversely, if no electron is observed, stringent bounds on lepton flavour violation would follow. To be precise, for the LHC we estimate that if no electrons are observed, the bound

$$
\left|U_{11}\right|^{2}+\left|U_{14}\right|^{2} \lesssim 8 \times 10^{-4} \quad \text { for } N_{\widetilde{\tau}}=3000,
$$

would follow at $90 \%$ confidence level. Analogously, for the LC, if no electrons are observed, the corresponding bound would be

$$
\left|U_{11}\right|^{2}+\left|U_{14}\right|^{2} \lesssim 8 \times 10^{-5} \quad \text { for } N_{\widetilde{\tau}}=30000,
$$

at $90 \%$ confidence level. Hence, mixing angles in the slepton sector as small as $\sim 3 \times$ $10^{-2}\left(9 \times 10^{-3}\right)$ could be probed at the $90 \%$ confidence level if $3 \times 10^{3}\left(3 \times 10^{4}\right)$ sleptons could be collected. This is the main result of this paper. 

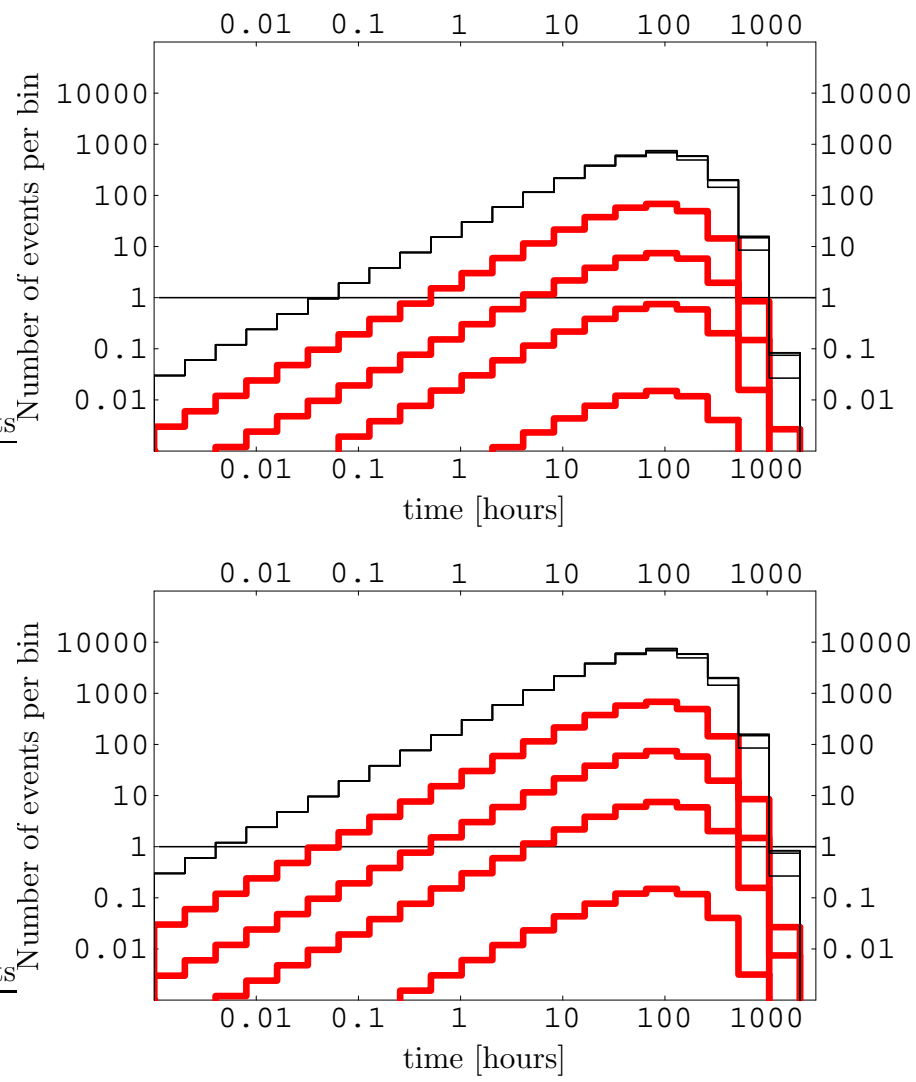

Figure 3: Number of electrons (thick red lines) and taus (thin black lines) per logarithmic time bin, $[t, 2 t]$, for the case in which only staus are present in the sample. In the upper plot we show a typical case for the LHC $N_{\tilde{\tau}}=3000$, while in the lower plot for a $e^{-} e^{-}$Linear Collider, $N_{\tilde{\tau}}=30000$. We take as representative decay rates $\Gamma\left(\widetilde{\tau} \rightarrow \tau \psi_{3 / 2}\right)=\Gamma\left(\widetilde{e} \rightarrow e \psi_{3 / 2}\right)=(100 \text { hours })^{-1}$. The different curves correspond to different amounts of lepton flavour violation: $\Gamma\left(\widetilde{\tau} \rightarrow e \psi_{3 / 2}\right) / \Gamma\left(\widetilde{\tau} \rightarrow \tau \psi_{3 / 2}\right)=$ $0.1,0.01,0.001$ and 0 from the top to the bottom. The number of taus hardly differs when lepton flavour violation is switched on. Note that the background from the tau decay (the lowest line) is negligible.

It is interesting to mention that when left-right mixing is not too large (in particular, for small or moderate $\tan \beta$ ), the NLSP consists mainly of a right-handed stau. Therefore, in their decays we would be probing flavour violation in the right-handed sector, which is a rather elusive sector. Lepton flavour violating processes generated radiatively, such as $\tau \rightarrow e \gamma$, are very insensitive to the right-handed sector, resulting in very poor constraints for the right-handed mixing angles [13]. In consequence, the tree-level flavour violating stau decays studied in this paper could offer a unique opportunity to probe this sector, if this scenario is realized in nature.

Let us discuss now the results in the cases where the three conditions above are simultaneously fulfilled. If there is no LFV, or the amount of LFV is so small that $\Gamma(\widetilde{e} \rightarrow \widetilde{\tau} e e) \ll \Gamma\left(\widetilde{e} \rightarrow e \psi_{3 / 2}\right)$, then selectrons decay mainly via $\widetilde{e} \rightarrow \widetilde{\tau} \bar{\nu}_{\tau} \nu_{e}$ or $\widetilde{e} \rightarrow e \psi_{3 / 2}$. Since the decay rates of those modes are very much suppressed, selectrons reach the stopperdetector before decaying, and two different scenarios could arise depending on the relative size of $\widetilde{e} \rightarrow \widetilde{\tau} \bar{\nu}_{\tau} \nu_{e}$ and $\widetilde{e} \rightarrow e \psi_{3 / 2}$. 

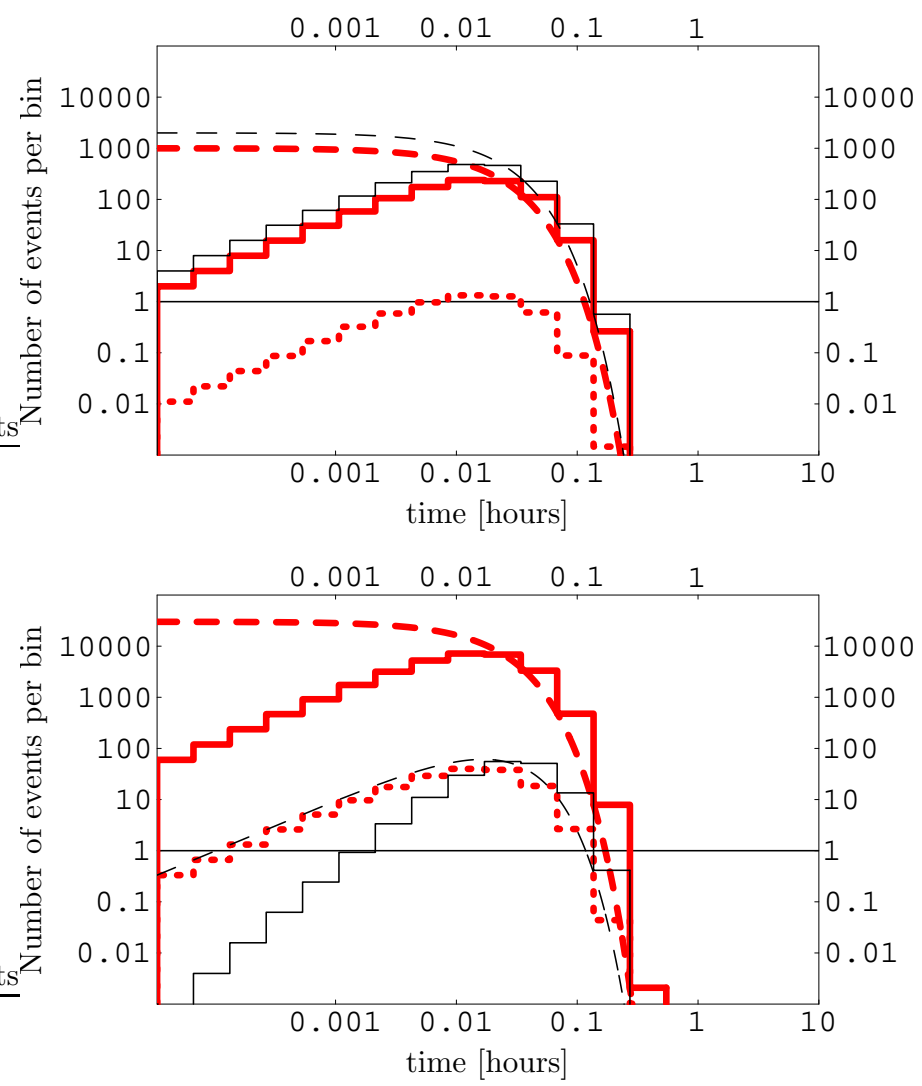

Figure 4: Number of events per logarithmic time bin, $[t, 2 t]$, for a typical case in which the sample consists of both selectrons and staus, and $\Gamma\left(\widetilde{e} \rightarrow \widetilde{\tau} \nu_{e} \bar{\nu}_{\tau}\right) \ll \Gamma\left(\widetilde{e} \rightarrow e \psi_{3 / 2}\right)$. In the upper plot we show the number of events for $N_{\widetilde{\tau}}$ (init.) $=2000$ and $N_{\widetilde{e}}$ (init.) $=1000$, while in the lower plot $N_{\widetilde{\tau}}$ (init.) $=0, N_{\widetilde{e}}$ (init. $)=30000$. The different decay rates are $\Gamma\left(\widetilde{\tau} \rightarrow \tau \psi_{3 / 2}\right)=\Gamma\left(\widetilde{e} \rightarrow e \psi_{3 / 2}\right)=$ $(1 \mathrm{~min})^{-1}$ and $\Gamma\left(\widetilde{e} \rightarrow \widetilde{\tau} \nu_{e} \bar{\nu}_{\tau}\right)=(3 \text { hours })^{-1}$. The thick red and the thin black histograms represent the number of electrons and taus, respectively, whereas the dotted red histogram represent the (invisible) events $\widetilde{e} \rightarrow \widetilde{\tau}$. We also show the number of selectron (thick red dashed line) and stau (thin black dashed line).

If $\Gamma\left(\widetilde{e} \rightarrow \widetilde{\tau} \nu_{e} \bar{\nu}_{\tau}\right) \ll \Gamma\left(\widetilde{e} \rightarrow e \psi_{3 / 2}\right)$, the conversion of selectrons into staus is very slow, and the dominant decay channel of the selectrons is the gravitational one, with the same lifetime as the staus. Therefore, in the detector we would observe electrons and taus, presenting a temporal distribution with peaks at very similar times. A clear signature for this scenario would be the detection of a large amount of electrons in the detector, as many as selectrons were produced initially, so this case is clearly distinguishable from the other ones. In figure 1 we show the electron and tau distribution for typical parameters corresponding to this scenario. If this possibility is realized in nature, it would be possible to measure the stau and the selectron lifetimes from the events $\widetilde{\tau} \rightarrow \tau \psi_{3 / 2}$ and $\widetilde{e} \rightarrow e \psi_{3 / 2}$, and accordingly test the universality of the gravitino coupling.

On the other hand, it could happen that $\Gamma\left(\widetilde{e} \rightarrow \widetilde{\tau} \bar{\nu}_{\tau} \nu_{e}\right) \gg \Gamma\left(\widetilde{e} \rightarrow e \psi_{3 / 2}\right)$ (while preserving the condition $\left.\Gamma\left(\widetilde{e} \rightarrow \widetilde{\tau} \bar{\nu}_{\tau} \nu_{e}\right) \lesssim N_{\widetilde{e}} \Gamma\left(\widetilde{e} \rightarrow e \psi_{3 / 2}\right)\right)$. In this case, one could see both electron events and tau events in the detector, with the peaks of their temporal 

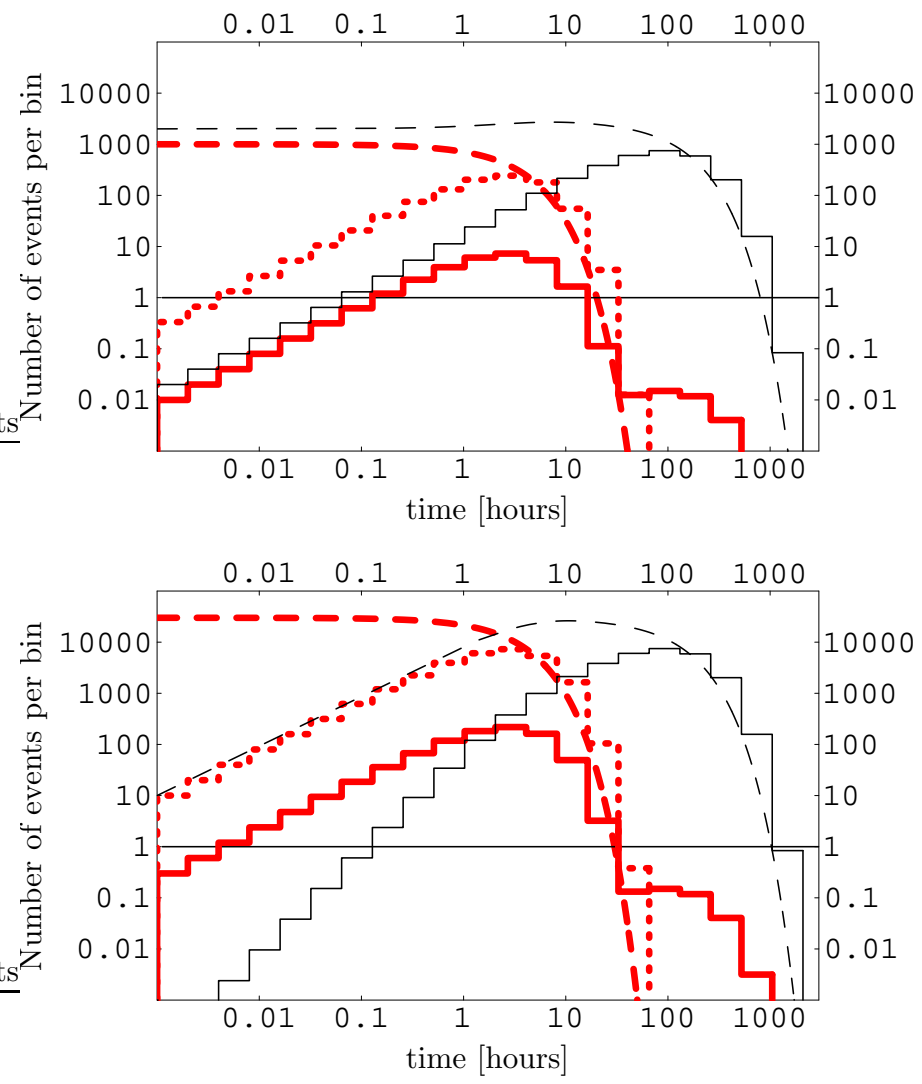

Figure 5: The same as figure $\pi$ but for a case with $\Gamma\left(\widetilde{e} \rightarrow \widetilde{\tau} \nu_{e} \bar{\nu}_{\tau}\right) \gg \Gamma\left(\widetilde{e} \rightarrow e \psi_{3 / 2}\right)$. We take as representative decay rates $\Gamma\left(\widetilde{\tau} \rightarrow \tau \psi_{3 / 2}\right)=\Gamma\left(\widetilde{e} \rightarrow e \psi_{3 / 2}\right)=(100 \text { hours })^{-1}$ and $\Gamma\left(\widetilde{e} \rightarrow \widetilde{\tau} \nu_{e} \bar{\nu}_{\tau}\right)=$ $(3 \text { hours })^{-1}$.

distribution occurring at different times, corresponding to the two different lifetimes $\tau_{\widetilde{\tau}}$ and $\tau_{\widetilde{e}}$. This case is potentially dangerous for the study of lepton flavour violation. In contrast to the case with $\Gamma\left(\widetilde{e} \rightarrow \widetilde{\tau} \nu_{e} \bar{\nu}_{\tau}\right) \ll \Gamma\left(\widetilde{e} \rightarrow e \psi_{3 / 2}\right)$, now an important fraction of selectrons could decay into staus, so the number of electron events in the detector could be small. Therefore, just from counting the total number of events, this scenario could be mistaken with the scenario with small flavour violation, that would also yield a small number of electrons in the detector. Nevertheless, it could be possible to discriminate this scenario from the one with small flavour violation by looking at the temporal distribution of events. This is shown in figure 5 for typical parameters corresponding to this scenario. Comparing with figure 3, it is apparent that in this case the peak of the electron distribution appears at earlier times, and clearly displaced from the peak of the tau distribution. Another possibility to discriminate between these two scenarios would be searching the two electrons emitted in the flavour violating selectron decay mediated by neutralinos, $\widetilde{e} \rightarrow \widetilde{\tau} e e$. Notice that these decays could offer an alternative method for detecting flavour violation, although the analysis of the backgrounds is much more involved. A further study along this lines would be certainly interesting, but lies beyond the scope of this paper. Detailed studies of lepton flavour violation via $\widetilde{e} \rightarrow \widetilde{\tau} e e$ will be presented elsewhere. 


\section{Conclusions}

In this paper we have discussed the prospects for observing lepton flavour violation in the decay of the lightest charged slepton, in scenarios where the gravitino is the lightest superparticle. In this class of scenarios, renormalization group analyses of the masses of the superparticles and different cosmological arguments, point to the possibility that the lightest charged slepton, generically a right-handed stau, is the next-to-lightest superparticle. Therefore, it can only decay gravitationally into charged leptons and gravitinos, with very long lifetimes due to the gravitational suppression of the interaction. This would allow the collection of staus and the clean observation of their decay products. The normal (flavour conserving) decay mode of staus would yield taus, in consequence, the observation of electrons and muons in the detector would be an indication for lepton flavour violation.

We have studied the different sources of backgrounds and identified the potentially most disturbing background, namely the possibility that selectrons are as long lived as staus, so that the electrons from their flavour conserving decays could be mistaken for electrons from the flavour violating stau decay. Nevertheless, we have shown that if flavour violation is large enough to be observed in these experiments, the selectron decay channel $\widetilde{e} \rightarrow \widetilde{\tau} e e$ is very efficient. Therefore, selectrons are never long lived enough to represent an important source of background. We have remarked the interesting double role that flavour violation plays in this experiment, both as object of investigation and as crucial ingredient for the success of the experiment itself.

We have estimated that in the LHC or the future Linear Collider it would be possible to probe mixing angles in the slepton sector down to the level of $\sim 3 \times 10^{-2}\left(9 \times 10^{-3}\right)$ at $90 \%$ confidence level if $3 \times 10^{3}\left(3 \times 10^{4}\right)$ staus could be collected. It is important to stress that this experiment probes directly flavour violation in the right-handed sector, which is a sector rather difficult to constrain from radiative rare decays, such as $\tau \rightarrow e \gamma$.

\section{Acknowledgments}

We would like to thank the organizers of SUSY04, Tsukuba, Japan, June 17-23, 2004, where this work has been initiated. We are grateful to Concha González-García and Mihoko M. Nojiri for helpful discussions.

\section{A. Electron spectrum from tau decay}

We would like to review here the electron spectrum coming from the decay of taus in flight 15] (the muon spectrum is completely analogous). We assume that the NLSPs are stopped in the laboratory frame, so the taus from NLSP decay have an energy $E_{\tau}=$ $\left(m_{\tilde{\tau}}^{2}+m_{\tau}^{2}-m_{3 / 2}^{2}\right) /\left(2 m_{\tilde{\tau}}\right)$. Generically, and as long as $m_{\tilde{\tau}}-m_{3 / 2} \gg m_{\tau}$, the outgoing leptons from NLSP decay are ultrarelativistic. Therefore, the cut-off of the energy spectrum of the electrons from tau decay, $E_{\tau}$, is very similar to the energy of the electrons from the LFV stau decay, $E_{0}$, defined after eq. (3.5). 
Taus decay into electrons with a continuous spectrum, whose peak lies at

$$
E_{\mathrm{peak}}=\frac{1}{2}\left(E_{\tau}-\sqrt{E_{\tau}^{2}-m_{\tau}^{2}}\right) \simeq \frac{m_{\tau}^{2}}{4 E_{\tau}}
$$

where we have used $E_{\tau} \gg m_{\tau}$ and we have neglected the mass of the electron. It is important to note that as the taus become ultrarelativistic, the peak of the electron spectrum is shifted to low energies. Therefore, the number of electrons with energies close to $E_{0}$ coming from the tau decay is tiny, which reduces enormously the background for the lepton flavour violating decay. For energies smaller than $E_{\text {peak }}$, the electron spectrum is described by

$$
\frac{d \Gamma}{d E_{e}}=\frac{G_{F}^{2}}{18 \pi^{3}} \frac{\sqrt{E_{e}^{2}-m_{e}^{2}}}{E_{\tau}}\left[2 E_{e}^{2}\left(m_{\tau}^{2}-4 E_{\tau}^{2}\right)+\frac{9}{2} E_{e} E_{\tau}\left(m_{e}^{2}+m_{\tau}^{2}\right)+m_{e}^{2}\left(2 E_{\tau}^{2}-5 m_{\tau}^{2}\right)\right]
$$

while for energies larger than $E_{\text {peak }}$, the corresponding expression reads

$$
\frac{d \Gamma}{d E_{e}}=\frac{G_{F}^{2}}{192 \pi^{3}} \frac{\Omega}{E_{\tau} \sqrt{E_{\tau}^{2}-m_{\tau}^{2}}}\left[-\frac{4}{3} \Omega^{2}+\Omega\left(m_{e}^{2}+m_{\tau}^{2}\right)+2\left(m_{e}^{2}-m_{\tau}^{2}\right)^{2}\right],
$$

where $\Omega=m_{\tau}^{2}+m_{e}^{2}-2 E_{\tau} E_{e}+2 \sqrt{E_{\tau}^{2}-m_{\tau}^{2}} \sqrt{E_{e}^{2}-m_{e}^{2}}$ and $E_{e}$ is the energy of the outgoing electron in the laboratory frame.

In the region of interest, the region close to the cut-off $E_{0}$, this formula is simplified to:

$$
\frac{d \Gamma}{d E_{e}} \simeq \frac{G_{F}^{2}}{192 \pi^{3}} \frac{m_{\tau}^{6}}{E_{\tau}^{2}}\left[\frac{5}{3}-3\left(\frac{E_{e}}{E_{\tau}}\right)^{2}+\frac{4}{3}\left(\frac{E_{e}}{E_{\tau}}\right)^{3}\right],
$$

where we have neglected the mass of the outgoing electron, and we have taken the limit $E_{\tau} \gg m_{\tau}$. It is convenient to express the energy of the electrons relative to the cut-off energy, defining $x=E_{e} / E_{\tau}$. With this definition, $0<x \lesssim 1-m_{\tau}^{2} / 4 E_{\tau}^{2}$. Therefore, the energy spectrum in the region of interest can be rewritten as:

$$
\frac{d \Gamma}{d x} \simeq \frac{G_{F}^{2}}{192 \pi^{3}} \frac{m_{\tau}^{6}}{E_{\tau}} f(x),
$$

where $f(x) \equiv \frac{5}{3}-3 x^{2}+\frac{4}{3} x^{3}$ is the fraction of electrons with energy $x$.

We are interested in electrons with energies close to the cut-off $E_{0}$, i.e. with energies in the range $1-\Delta x<x \lesssim 1-m_{\tau}^{2} / 4 E_{\tau}^{2}$, being $\Delta x \ll 1$. The number of electrons in this energy bin is:

$$
N_{e}^{L F C}=N_{\widetilde{\tau}} B R\left(\widetilde{\tau} \rightarrow \tau \psi_{3 / 2}\right) B R\left(\tau \rightarrow e \bar{\nu}_{e} \nu_{\tau}\right) \int_{1-\Delta x}^{1-\frac{m_{\tau}^{2}}{4 E_{\tau}^{2}}} d y \int_{0}^{1-\frac{m_{\tau}^{2}}{4 E_{\tau}^{2}}} d z f(z) \frac{e^{-\frac{(z-y)^{2}}{2 \sigma^{2}}}}{\sigma \sqrt{2 \pi}} .
$$

For a typical electromagnetic calorimeter, the energy resolution is $\sigma \simeq 10 \% / \sqrt{E(\mathrm{GeV})}$. Therefore, the fraction of electrons in that energy bin, that can be mistaken for electrons coming from the LFV stau decay is $N_{e}^{L F C} \simeq 2 \times 10^{-5} N_{\widetilde{\tau}}$ for $E_{\tau} \simeq 100 \mathrm{GeV}$. For the number of NLSPs that we are using to illustrate our results, the number of electrons from this source of background turns out to be negligible in most instances. 


\section{B. Probing $R$-parity violation in slepton NLSP scenarios}

In the body of the paper we have assumed that the $R$-parity is conserved. In this appendix we briefly discuss possible probe of R-parity violations via long-lived charged sleptons. Clearly, the long lifetime of the NLSP slepton leads to a severe bound on the $R$-parity violating couplings. Let us concentrate on the following trilinear $R$-parity violating operators in the superpotential

$$
W_{R p V}=\frac{1}{2} \lambda^{i j k} L_{i} L_{j} E_{k}^{c}+\lambda^{\prime i j k} L_{i} Q_{j} D_{k}^{c},
$$

and assume that the NLSP is mainly the lighter stau, $\widetilde{\tau}_{1}=\cos \theta_{\widetilde{\tau}} \widetilde{\tau}_{R}+\sin \theta_{\widetilde{\tau}} \widetilde{\tau}_{L}$. If we observe that the stau decays mainly into tau and gravitino (missing particle) with lifetime $\tau_{\tilde{\tau}}$, one obtains the following bounds

$$
\left|\lambda^{3 j k} \sin \theta_{\widetilde{\tau}}\right|^{2}, 2\left|\lambda^{i j 3} \cos \theta_{\widetilde{\tau}}\right|^{2}, 3\left|\lambda^{\prime 3 j k} \sin \theta_{\widetilde{\tau}}\right|^{2} \lesssim 10^{-30} \times\left(\frac{100 \mathrm{GeV}}{m_{\widetilde{\tau}}}\right)\left(\frac{100 \text { hours }}{\tau_{\widetilde{\tau}}}\right),
$$

where we have assumed that there is no cancellation between couplings. Note that the bounds range by orders of magnitude depending on the lifetime.

If on the contrary the $R$-parity violation would dominate the decay, the signals would be (i) for $\lambda^{\prime 3 j k}$ coupling; two jets with identical energies $E_{j e t}=E_{0}=m_{\widetilde{\tau}} / 2$, (ii) for $\lambda^{123}$; one lepton $(e$ or $\mu)$ with energy $E_{0}$ plus missing particle, with branching ratios being $B R\left(\widetilde{\tau} \rightarrow e \nu_{\mu}\right)=B R\left(\widetilde{\tau} \rightarrow \mu \nu_{e}\right)$, (iii) for $\lambda^{3 j k}(k \neq 3)$; one lepton $\left(l_{k}, k \neq 3\right)$ with energy $E_{0}$ plus missing particle, and (iv) for $\lambda^{3 j 3}$; one lepton $\left(\tau\right.$ or $\left.l_{j}, j \neq 3\right)$ with energy $E_{0}$ plus missing particle with $B R\left(\widetilde{\tau} \rightarrow \tau \nu_{j}\right) \sim B R\left(\widetilde{\tau} \rightarrow l_{j} \nu_{\tau}\right)$.

\section{References}

[1] T. Moroi, H. Murayama and M. Yamaguchi, Cosmological constraints on the light stable gravitino, Phys. Lett. B 303 (1993) 289;

M. Bolz, A. Brandenburg and W. Buchmuller, Thermal production of gravitinos, Nucl. Phys. B 606 (2001) 518 hep-ph/0012052.

[2] J.L. Feng, A. Rajaraman and F. Takayama, Superweakly-interacting massive particles, Phys. Rev. Lett. 91 (2003) 011302 hep-ph/0302215; Superwimp dark matter signals from the early universe, Phys. Rev. D 68 (2003) 063504 [hep-ph/0306024;

J.L. Feng, S.-f. Su and F. Takayama, Superwimp gravitino dark matter from slepton and sneutrino decays, Phys. Rev. D 70 (2004) 063514 hep-ph/0404198;

See also, T. Asaka, K. Hamaguchi and K. Suzuki, Cosmological gravitino problem in gauge mediated supersymmetry breaking models, Phys. Lett. B 490 (2000) 136 hep-ph/0005136.

[3] K. Hamaguchi, Y. Kuno, T. Nakaya and M.M. Nojiri, A study of late decaying charged particles at future colliders, Phys. Rev. D 70 (2004) 115007 hep-ph/0409248.

[4] J.L. Feng and B.T. Smith, Slepton trapping at the large hadron and international linear colliders, Phys. Rev. D 71 (2005) 015004 hep-ph/0409278.

[5] W. Beenakker, R. Hopker, M. Spira and P.M. Zerwas, Squark and gluino production at hadron colliders, Nucl. Phys. B 492 (1997) 51 hep-ph/9610490. 
[6] ECFA/DESY LC Physics Working Group collaboration, J.A. Aguilar-Saavedra et al., TESLA technical design report part iii: physics at an $e^{+} e^{-}$linear collider, hep-ph/0106315; ACFA Linear Collider Working Group collaboration, K. Abe et al., Particle physics experiments at $j l c$, hep-ph/0109166;

American Linear Collider Working Group collaboration, T. Abe et al., Linear collider physics resource book for snowmass 2001. 1: introduction, hep-ex/0106055.

[7] W. Buchmuller, K. Hamaguchi, M. Ratz and T. Yanagida, Supergravity at colliders, Phys. Lett. B 588 (2004) 90 hep-ph/0402179;

W. Buchmuller, K. Hamaguchi, M. Ratz and T. Yanagida, Gravitino and goldstino at colliders, hep-ph/0403203.

[8] N.V. Krasnikov, Search for flavor lepton number violation in slepton decays at LEP2 and NLC, Phys. Lett. B 388 (1996) 783 hep-ph/9511464;

N. Arkani-Hamed, H.-C. Cheng, J.L. Feng and L.J. Hall, Probing lepton flavor violation at future colliders, Phys. Rev. Lett. 77 (1996) 1937 hep-ph/9603431;

J. Hisano, M.M. Nojiri, Y. Shimizu and M. Tanaka, Lepton flavor violation in the left-handed slepton production at future lepton colliders, Phys. Rev. D 60 (1999) 055008

hep-ph/9808410;

K. Agashe and M. Graesser, Signals of supersymmetric lepton flavor violation at the LHC, Phys. Rev. D 61 (2000) 075008 hep-ph/9904422];

D. Nomura, Probing left-handed slepton flavor mixing at future lepton colliders, Phys. Rev. D 64 (2001) 075001 hep-ph/0004256;

M. Guchait, J. Kalinowski and P. Roy, Supersymmetric lepton flavor violation in a linear collider: the role of charginos, Eur. Phys. J. C 21 (2001) 163 hep-ph/0103161;

$\mathrm{W}$. Porod and W. Majerotto, Large lepton flavor violating signals in supersymmetric particle decays at future $e^{+} e^{-}$colliders, Phys. Rev. D 66 (2002) 015003 hep-ph/0201284;

F. Deppisch, H. Pas, A. Redelbach, R. Ruckl and Y. Shimizu, The SUSY seesaw model and lepton-flavor violation at a future electron positron linear collider, Phys. Rev. D 69 (2004) 054014 hep-ph/0310053.

[9] M. Drees, Intermediate scale symmetry breaking and the spectrum of super partners in superstring inspired supergravity models, Phys. Lett. B 181 (1986) 279.

[10] S. Ambrosanio, G.D. Kribs and S.P. Martin, Three-body decays of selectrons and smuons in low-energy supersymmetry breaking models, Nucl. Phys. B 516 (1998) 55 hep-ph/9710217.

[11] J. Wess, J. Bagger, Supersymmetry and supergravity, Princeton University Press, Princeton 1992.

[12] M. Fujii, M. Ibe and T. Yanagida, Upper bound on gluino mass from thermal leptogenesis, Phys. Lett. B 579 (2004) 6 hep-ph/0310142;

J.L. Feng, S. Su and F. Takayama, Supergravity with a gravitino lsp, Phys. Rev. D 70 (2004) 075019 hep-ph/0404231;

J.R. Ellis, K.A. Olive, Y. Santoso and V.C. Spanos, Prospects for sparticle discovery in variants of the MSSM, hep-ph/0408118;

L. Roszkowski and R. Ruiz de Austri, Gravitino dark matter in the CMSSM and implications for leptogenesis and the $L H C$, hep-ph/0408227.

[13] I. Masina and C.A. Savoy, Sleptonarium (constraints on the CP and flavour pattern of scalar lepton masses), Nucl. Phys. B 661 (2003) 365 hep-ph/0211283. 
[14] Particle Data Group collaboration, S. Eidelman et al., Review of particle physics, Phys. Lett. B $592(2004) 1$.

[15] J.J. Gomez-Cadenas and C.M. Gonzalez-Garcia, Can we improve the tau-neutrino mass limit from the decay tau $\rightarrow$ lepton anti-lepton-neutrino tau-neutrino?, Phys. Rev. D 39 (1989) 1370 . 